\title{
Key Technologies in Massive MIMO
}

\author{
Qiang $\mathrm{Hu}^{1}$, Meixiang $\mathrm{Zhang}^{1}$, and Renzheng $\mathrm{Gao}^{1}$ \\ ${ }^{1}$ College of Information Enginnering Yangzhou University, Yangzhou 225127, China
}

\begin{abstract}
The explosive growth of wireless data traffic in the future fifth generation mobile communication system $(5 \mathrm{G})$ has led researchers to develop new disruptive technologies. As an extension of traditional MIMO technology, massive MIMO can greatly improve the throughput rate and energy efficiency, and can effectively improve the link reliability and data transmission rate, which is an important research direction of $5 \mathrm{G}$ wireless communication. Massive MIMO technology is nearly three years to get a new technology of rapid development and it through a lot of increasing the number of antenna communication, using very duplex communication mode, make the system spectrum efficiency to an unprecedented height.
\end{abstract}

\section{Introduction}

In recent years, the growth of mobile data traffic has been increasing rapidly and is expected to reach 200 to 1,000 times by 2020 . At the same time, with the increasing energy consumption of the communication system, reducing the energy consumption of the system has gradually become one of the important goals of mobile communication development. The fourth generation mobile communication system (4G) is hard to meet people on the spectrum efficiency and power efficiency of higher demand, the future of the fifth generation mobile communication system $(5 \mathrm{G})$ need to be in network architecture, network protocols and new breakthrough in wireless transmission technology, etc, in order to achieve higher spectral efficiency and green dual goals of wireless communication [1][2].

Multiple-input multiple-output (MIMO), also known as the multiple antenna technology, through the communication link of sending and receiving set up multiple antennas at both ends and make full use of space resources, can provide diversity gain in order to enhance the system reliability, provide multiplexing gain in order to increase the frequency spectrum of the system efficiency, provide array gain in order to improve the power efficiency of the system, and in the past 20 years has been one of the mainstream technology in the field of wireless communications. Currently, MIMO technology has been adopted by The 3rd Generation Partnership Project (3GPP), 3GPP's LTE/LTE-Advanced and The Institute of Electrical and Electronics Engineers's WiMAX and other 4G standards [3]. However, the existing 4G system base stations have fewer antennas (generally no more than 8), and MIMO performance gains are severely limited.

In response to the lack of traditional MIMO technology, Marzetta of bell laboratories in the US proposed the concept of massive MIMO in 2010 [4]. In a massive MIMO system,

·Corresponding author: huqiang1210hq@163.com 
the base station is configured with tens to hundreds of antennas, which is an order of magnitude larger than that of the traditional MIMO system. The base station makes full use of the space freedom of the system and serves a number of users at the same time. Study found that when a village on the number of base station antenna becomes infinite, different user channel will tend to be orthogonal, additive white gaussian noise and small scale fading, all negative effects can be neglected, only limited to single user throughput is the same in the other community pilot sequences of interference users, system throughput than traditional MIMO system more than one order of magnitude [5][6]. The evolution of traditional MIMO to massive MIMO is a process from quantitative to qualitative change. Due to the large number of base station antenna MIMO and air separation users than traditional MIMO is orders of magnitude increases, both in the wireless communication on the basic principles and specific methods had different both in common, it provides researchers with contains many high research value of "gold mining and metallurgy" [7]. In recent years, the basic theory of massive MIMO, channel measurement and modeling, channel information acquisition, wireless transmission, experiment and testing have yielded fruitful results.

The structure of this paper is as follows: the second section analyzes the MIMO technology standardization process in detail, and the third section analyzes the technical advantages of massive MIMO and the main application scenarios compared with the traditional MIMO technology. The fourth and fifth sections are introduced from the point of view of channel measurement and channel modeling, and the channel estimation techniques for massive MIMO systems are analyzed in detail. Then precoding technology and signal detection technology are introduced. The eighth section illustrates the technical challenges faced by massive MIMO technology today. At the end of this paper, the summary of this paper is made.

\section{Traditional MIMO technology}

MIMO technology was first established in 1908 by Marconi, it by the sender and the receiver are equipped with multiple antennas to improve the capacity of communication system, system data transmission rate and transmission reliability. MIMO technology is one of the key technologies of the third generation mobile communication system ( $3 \mathrm{G})$ and $4 \mathrm{G}$ communication systems.

\subsection{Single user MIMO technology}

The earliest point to point MIMO communication transmission technology, that is, single user MIMO (SU-MIMO), SU-MIMO using multiple antennas at both the transmitter and receiver to carry out wireless communication. In the actual system, if the multiple lines between the transmitter or the receiver are not related to each other, the distance between the adjacent antennas must be greater than the carrier wavelength. For handheld mobile terminals, physical size is limited, placing multiple antennas and uncorrelated antennas is very difficult, which limits the actual gain of SU-MIMO system. The basic model of SU-MIMO is shown in Figure 1.

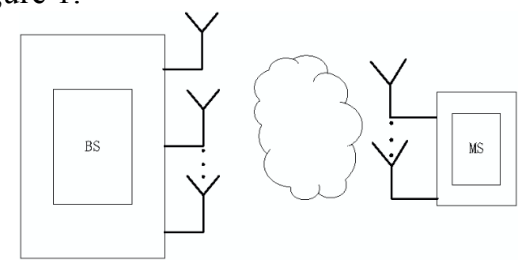

Fig. 1. The basic model of SU-MIMO. 


\subsection{Multiple user MIMO technology}

The actual wireless communication system tends to be a base station (BS) communication with multiple mobile terminals (MS), so a multiple user MIMO (MU-MIMO) appears [8]. In more community MU-MIMO case, inside the village interference can be through the night, frequency division and reduce or eliminate the code classification and simple technology, but the interference between zones must adopt some more complex technology to reduce or eliminate, such as using Maximum Likelihood Multi User Detection(MLMUD) in the uplink or in the downlink using Dirty Paper Coding (DPC) technology. Inter cell interference leads to bottlenecks in MU-MIMO and cooperative MU-MIMO that limit spectrum and power efficiency enhancement. The basic model of MU-MIMO is shown in Figure 2.
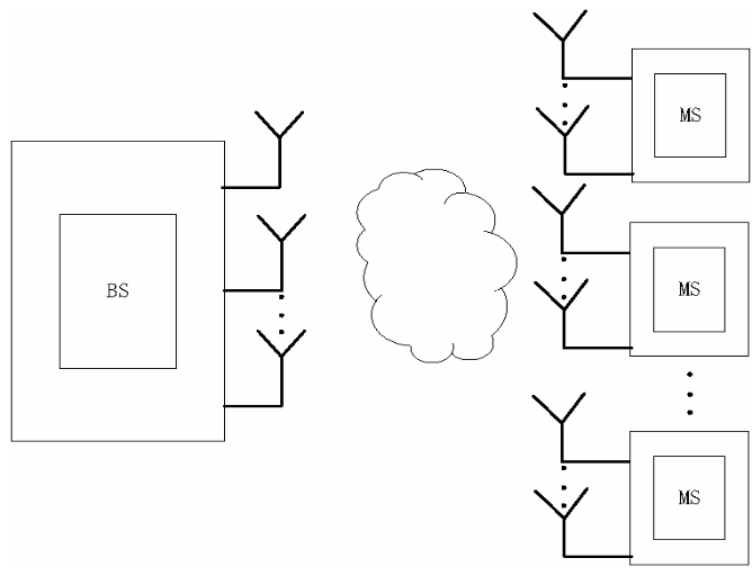

Fig. 2. The basic model of MU-MIMO

\subsection{Standardization of MIMO Technology}

The MIMO technology standardization process in LTE/LTE-A is shown in Table 1.

Table 1. MIMO technology standardization process.

\begin{tabular}{|c|c|c|}
\hline Standard & MIMO Technology & Characteristic \\
\hline Rel-8 & $\begin{array}{l}\text { Transmit diversity } \\
\text { Space-division } \\
\text { multiplexing } \\
\text { Beamforming } \\
\text { MU-MIMO }\end{array}$ & $\begin{array}{l}\text { Supports up to } 4 \text { layers of transmission only } \\
\text { support single-layer transmission up to two } \\
\text { rank1Ue }\end{array}$ \\
\hline Rel-9 & $\begin{array}{l}\text { Dual stream } \\
\text { beamforming }\end{array}$ & $\begin{array}{l}\text { SU / MU flexible switch up to } 4 \text { data } \\
\text { streams(Up to } 2 \text { layers per UE)using } \\
\text { non-codebook transmission support } \\
\text { opportunity channel reciprocity feedback }\end{array}$ \\
\hline \multirow[t]{2}{*}{ Rel-10 } & High-order MIMO & $\begin{array}{l}\text { Supports up to } 8 \text { layers of transmission High } \\
\text { Precision Feedback Based on Two Level } \\
\text { Multi - granularity Codebook }\end{array}$ \\
\hline & Uplink MIMO & Supports up to 4 layers of transmission \\
\hline $\begin{array}{l}\text { Rel-11 } \\
\text { Rel-12 } \\
\text { Rel-13 }\end{array}$ & $\begin{array}{l}\text { CoMP } \\
\text { CoMP/3DMIMO } \\
\text { 3D MIMO }\end{array}$ & $\begin{array}{l}\text { Multi-cell support for collaborative MIMO } \\
\text { Multi-cell collaborative MIMO / 3D } \\
\text { Expand to three-dimensional (3D) antenna } \\
\text { array }\end{array}$ \\
\hline
\end{tabular}




\section{Massive MIMO technology}

In 2010, the Bell laboratory scientist Marzetta proposed the concept of massive MIMO in the multi-cell and Time Division Duplexing (TDD) scenarios, thus discovering some different features of the single cell and the limited number of antennas [4]. Massive MIMO technology refers to the large number of antennas deployed by the base station, usually one hundred or hundreds of antennas [9], which is increased by several orders of magnitude over the existing communication system. More than one user is served at the same time frequency resource, and the mobile terminal generally uses a single antenna reception [10]. The basic model of massive MIMO is shown in Figure 3.

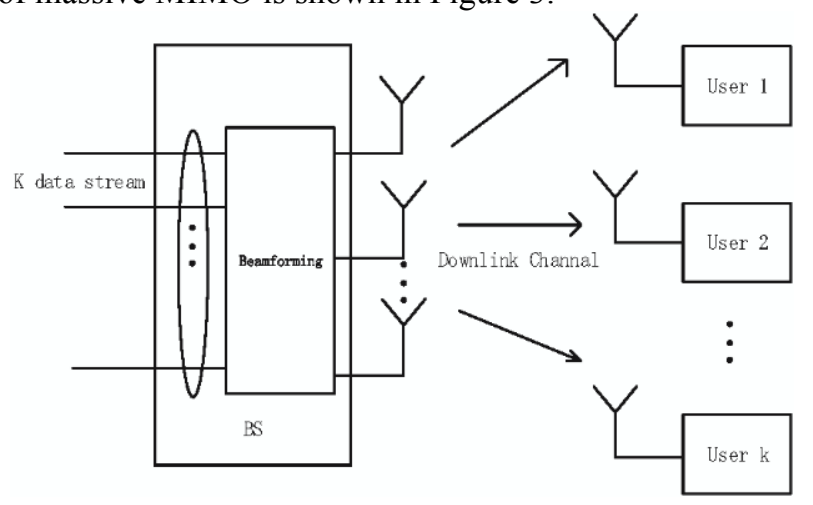

Fig. 3. The basic model of massive MIMO.

\subsection{Technical advantages of massive MIMO}

The main advantages of massive MIMO technology are as follows:

1) The power of the antenna is very low: Under ideal conditions, the transmitting power of each antenna is inversely proportional to the number of antennas, and under certain conditions of transmitting signal-to-noise ratio (SNR), the total emission power is also inversely proportional to the number of antennas. Therefore, the emission power required for each antenna is inversely proportional to the square of the number of antennas. This can effectively reduce the power consumed in massive MIMO applications.

2) Channel "hardening": The number antennas tends to infinity, the channel matrix of random matrix theory may be used for analysis of channel matrix singular value would be known gradually distribution, and channel vector will tend to be orthogonal, the simplest signal processing method is optimal.

3) The effects of thermal noise and small scale decline are eliminated: Using linear signal processing method, the thermal noise and small scale fading effect the performance of the system increases with the decrease in the number of antennas, and the effect of thermal noise and small scale fading are negligible in comparison with interference between zones.

4) Spatial resolution enhancement: In massive MIMO systems, with the increase of base station antennas, beamforming can transmit the transmitted signals to one point of the space, that is, the base station can accurately distinguish each user, thus improving the spatial resolution [11].

\subsection{Massive MIMO comparison with traditional MIMO performance}

In 3GPP, MIMO technology is generally evolving along SU-MIMO, MU-MIMO to network MIMO development. Compared with the traditional MIMO technology, the 
performance of massive MIMO technology is reflected in many aspects. Table 2 gives the performance comparison between traditional MIMO and massive MIMO.

Table 2. Traditional MIMO and massive MIMO performance comparison

\begin{tabular}{ccc}
\hline Technical content & $\begin{array}{c}\text { Traditional } \\
\text { MIMO }\end{array}$ & Massive MIMO \\
\hline The number of antennas & $\leq 8$ & $\leq 100$ \\
\hline Channel angle value & uncertain & As the matrix field grows to form a deterministic function \\
\hline Channel matrix & $\begin{array}{c}\text { Low } \\
\text { demand }\end{array}$ & High demand \\
\hline Channel capacity & Low & High \\
\hline Diversity gain & Low & High \\
\hline Technical content & Traditional MIMO & Massive MIMO \\
\hline Link stability & Low & High \\
\hline To resist noise & Low & High \\
\hline Array resolution & Low & High \\
\hline Antenna correlation & Low & High \\
\hline Coupling & Low & High \\
\hline SER & High & Low \\
\hline Pilot pollution & no & yes \\
\hline
\end{tabular}

\subsection{Massive MIMO application scenarios}

The application scene of the massive multi-antenna MIMO technology in the fifth generation mobile communication system is shown in Figure 4. The two cell of macro cell and micro cell coexist in the application scenario of $5 \mathrm{G}$ massive antenna array. The network is mainly divided into two types: isomorphism and heterogeneous network, and scenes are divided into indoor scenes and outdoor scenes. According to the test related literature, about seventy percent of the mobile communication system on the land is from indoor. Therefore, massive MIMO channels can be divided into micro cell base stations, indoor or outdoor users, macro cell base stations for indoor or outdoor users. At the same time, the micro cell can be used as the relay base station to transmit information, and the channel can be divided into the macro cell base station and the micro cell base station. The number of antennas in the base station can be increased infinitely, and the number of mobile users in the cell can also be increased [12].

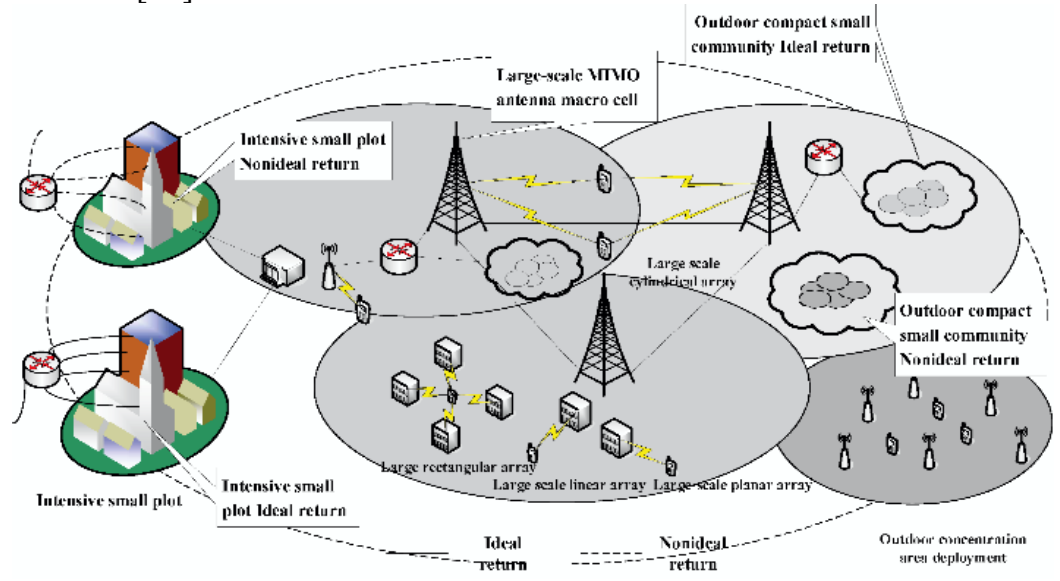

Fig. 4. Application scenarios of massive multi-antenna MIMO technology. 


\section{Measurement and modeling of massive MIMO channels}

When the wireless transmission conditions are ideal, the channel vectors of the transmitter and receiver are gradually orthogonal due to the increase of the large number of MIMO system antennas. The following main research and analysis of massive MIMO channel measurement and modeling.

\subsection{Measurement of massive MIMO channel}

Through the channel measurement in the actual wireless communication environment, the theoretical channel model of the massive MIMO system can be effectively verified. The performance of the whole communication system is improved by measuring the actual channel. And the perfect result analyzer provides an effective and intuitive tool for network performance analysis.

1) The distributed MIMO channel is measured under 2.6GHZ microcellular conditions : In order to study the crosscorrelation characteristics of large-scale fading of distributed MIMO consisting of four base stations and a mobile station between different base station links, the channel in $2.6 \mathrm{GHZ}$ microcell environment has been measured in the previous literature [6]. In these four base stations, three base stations have deployed four groups of highly polarized antenna units. Another base station has deployed only one antenna unit. The mobile station has deployed a cylindrical uniform array consisting of 64 groups of dual polarized antenna units. By using the above measurement method, we can get the large scale channel fading at different locations by analyzing the large-scale fading and cross-correlation characteristics between different base station links [13].

2) Measurement method of unit 128 linear array: The literature[7]considers 26 line-of-sight communication users, 10 non-line-of-sight communication users, the system working in $2.6 \mathrm{HZ}$, and the base station equipped with 128 unit linear arrays. In addition, the antenna array length and the antenna spacing are set to $7.3 \mathrm{~m}$ and half wavelengths respectively. In this paper, the massive MIMO channel is studied in the above scenario, and the Rciean $\mathrm{K}$ factor, the received power value of the array, the antenna correlation and the eigenvalue distribution are analyzed. The results show that due to the presence of some invisible scattering and the variation of the scattering power value, the propagation channel of large antenna arrays can not be regarded as a generalized stationary process. However, near-field effect and non-stationarity on array help to eliminate the correlation among users, thus providing a good, stable and low interference channel condition.

\subsection{Massive MIMO channel modeling}

Because of the rapid development of 5G technology, massive MIMO channel modeling has shown some new features. For example, spherical waves need to be replaced by a plane wave at the base station terminal large array antenna deployment, and the channel energy is concentrated in a limited space [14]. The channel is no longer independent and distributed. With the ever-increasing array of base station antennas, only different antenna units can see different scatterers and characterize the decline with non-static characteristics.

\section{Channel status information acquisition technology}

Bell laboratory, put forward the massive transmission scheme of MIMO system TDD mode, namely in the number of users equipped with single antenna is far less than the number of base station antenna assumptions, by receiving pilot signal to the target base station, 
according to the uplink channel estimation value of the CSI, reciprocity, TDD using TDD system has link with uplink CSI conjugate transpose down link of CSI. To transmission of uplink signal detection and descending precoding, the number of users in the village gains momentum, the pilot channel estimation cost will increase, especially in the high-speed mobile communications, pilot overhead will occupy most of the time and frequency resource. Therefore, it is of great practical value to study the massive MIMO CSI technology when TDD transmission mode pilot frequency is limited. Compared to Frequency Division Duplexing (FDD) mode, TDD mode can provide more ideal methods for obtaining CSI [15].

\section{Massive MIMO precoding technology}

Studies have shown that massive MIMO precoding technology plays a critical role in the bottleneck of breaking down the system's downward capacity. Nowadays, in massive MIMO downlink transmission, the purpose of using a large number of transmitter signal processing technology is to transform the complexity of massive MIMO system from the terminal side to the base station side. At present, there are mainly linear and nonlinear precoding algorithms, which are composed of zero forcing precoding (ZF), matched filtering precoding (MF) and block diagonalization (BD). The nonlinearity includes DPC, auxiliary grid method, vector perturbation (VP) and so on.

\subsection{Linear Precoding}

The linear and non-linear precoding technique has made extensive research [16], makes the massive MIMO precoding technology can reduce the system performance is affected by the pilot pollution, the following typical linear precoding algorithm will be introduced.

1) ZF precoding: In the literature [10], ZF precoding adopts the channel parameters replaced by pseudo inverse matrix [17]. In the literature [11], when meet the ratio of base station antenna number $\mathrm{M}$ and the terminal number $\mathrm{K}$ is constant, by increasing $\mathrm{M}$ and $\mathrm{K}$ at the same time, the matrix can be trace $\left\{\left(G^{H} G\right)^{-1}\right\}$ converges to $1 /(\propto-1)$ in which $A^{H}$ represent the Hermite matrix $A$ conjugate transpose.

2) MF precoding: The inverse calculation of $\mathrm{KxK}$ dimension matrix in $\mathrm{ZF}$ precoding will increase the complexity of the algorithm. In the massive MIMO, $\left(G^{H} G\right) / M$ gradually approach the identity matrix, and by simplifying the matrix to solve the inverse operation, the performance of the ZF precoding tends to MF precoding performance. With the expansion of the antenna array, the MF precoding matrix will be infinitely close to $\mathrm{ZF}$.

3) MMSE Precoding: In the multi-cell massive MIMO system, the assignment problem of the training sequence should be considered when the pre coding scheme is designed. The minimum mean squareerror estimation precoding (MMSE) scheme proposed in the literature [12] can reduce the pollution of pilots. Compared with the single cell scenario, the MMSE precoding matrix $A_{l}^{\mathrm{opt}}$ is obtained from the optimal solution of the objective function. In this paper, the target function is set up mainly by the mean square interference between the signal both received by the user in the same community and the user in the cross cell.

\subsection{Nonlinear Precoding}

Nonlinear precoding is by VP, DPC and auxiliary network method, etc, in the cell in the M and $\mathrm{K}$ is not especially large, non-linear precoding can show some advantages. In literature [15], the expression of SNR approximation in the VP of complete CSI is mentioned. 


\section{Massive MIMO system signal detection algorithm}

The station will be time-frequency resources among different users, to a large number of users to provide services in massive MIMO system in multi-cell multi-user terminal in the area of the transmitted signal is sent to the base station at the same time, the base station can by the spatial signature of the uplink signal received for testing.

\subsection{Linear detection}

When the base station is equipped with a large antenna array, if we can satisfy the condition of low SNR, the performance of the MRC receiver can achieve optimal linear receiver (OLR) performance. However, when the SNR is below OLR, the performance of OLR will be optimized compared with the typical MMSE receiver system when the interference is relatively large.

\subsection{Nonlinear detection}

1) Algorithm based on tree structure (TB): Sphere decoding (SD) is a typical nonlinear detection algorithm, SD is a maximal likelihood (ML) decoder [18]. SD algorithm shortcoming is only considering the specific radius point, in order to find any signaling point, to increase the radius, the existing of low complexity of TB in only some of the most valuable nodes are added can effectively reduce the complexity of the search.

2) Random Step (RS) method: The principle of this algorithm is: select an initial vector, evaluate its peripheral vector $N_{N e i g h}$ needs MSE as the condition, and select MSE as the minimum vector, repeat the above procedure $N_{i t e r}$.

\section{The technical challenges of massive MIMO}

Massive MIMO technology is one of the most promising technologies in mobile communication. However, from the perspective of system design and engineering implementation, there are still many key technical problems that need to be solved effectively.

\subsection{Channel model}

All wireless communication systems need to specify a corresponding system model to be used as a basis for performance evaluation and comparison. Considering that the array aperture of the massive MIMO is limited, the traditional linear array is no longer applicable and may need to be extended to the antenna array in three-dimensional space. There are few theoretical modeling and practical modeling of massive MIMO channels, and there is no widely accepted channel model.

\subsection{Channel information accuracy and feedback overhead}

Massive MIMO antennas require high precision CSI, and channel estimation accuracy, delay, and huge feedback overhead and processing will be the key factors to gain a better gain. The changing speed of the channel, the complexity of the coverage environment, the interference intensity of the cellular signal and the speed of the feedback information are also the key factors that affect the effect and successful deployment of massive MIMO. 


\subsection{Control broadcast channel design and algorithmic stability}

Although there has been a great deal of research on massive MIMO, it has not enough practical experience in the joint design with control broadcast channel and the stability of each algorithm in the actual complex environment. Therefore, it is inevitable for a certain time scale deployment and running in period.

\subsection{Resource scheduling}

In order to improve the capacity of the system, in the massive MIMO transmission, it is necessary to pair the antenna with the user to form a virtual MIMO channel. This process consists of grouping between users and the selection of antennas at the base station. At the same time, the allocation of spectrum resources needs to be considered when the number of users is relatively large.

\subsection{Active integrated antenna}

Due to the need of beamforming, the antenna design needs to combine the active circuit with the antenna to form a highly integrated active antenna system. It challenges the structure, size, weight, heat dissipation, cascade and maintenance of the antenna system. Especially in the aspect of antenna sharing, massive MIMO cannot share antennas with $2 \mathrm{G}$, $3 \mathrm{G}$ and existing systems. Under the increasingly tense antenna resources, there is a certain risk that the antenna form can be successfully commercialized.

\section{Conclusion}

Massive MIMO technology is considered to be a key technology in the future 5G. It has a significant effect on the channel capacity, energy efficiency and spectrum efficiency of wireless communication systems. This paper analyzes the standardization process of MIMO technology in detail, and the performance advantages of massive MIMO compared with traditional MIMO. On this basis, we analyzed and compared the application scenarios, channel measurement and channel modeling of massive MIMO, and analyzed the channel estimation technology, precoding technology and signal detection technology of massive MIMO system. In the future, due to the higher requirements of massive MIMO on hardware complexity, the presence of pilot pollution limits the improvement of system performance, and there are still many challenges to be completed.

\section{References}

1. CHIHLIN I,ROWELL C,HAN S F., "Toward green and soft: a 5G perspective", IEEE Communication Magazine, vol. 52, pp. 66-73,( 2014).

2. HUH HCAIRE GPAPADOPOULOS H C, "massive mimo spectral efficiency with a Not-sołLarge Number of Antennas", IEEE Transactions on Wireless Communications, vol. 11, no. 9, pp. 3266-3239, (2011).

3. S.-Y. Lien and K.-C. Chen, "Massive access management for QoS guarantees in 3GPP machine-to-machine communications," IEEE Commun.Lett., vol. 15, no. 3, pp. 311-313,(2011).

4. FOSCHINI G., "Layered space-time architecture for wireless communication in a fading environment when using multi-element antennas," Bell Labs Technical Journal, vol. 8, pp. 41-59, (1996). 
5. D. Gesbert, M. Kountouris, R. W. Heath, C.-B. Chae, and T. Salzer,"Shifting the MIMO paradigm: From single-user to multiuser communications," IEEE Signal Process. Mag., vol. 24, no. 5, pp. 36-46, (2007).

6. MARZETTA T L., "Noncooperative cellular wireless with unlimited numbers of base station antennas", IEEE Transactions on Wireless Communications, vol. 9, no. 11, pp. 3590-3600, (2010).

7. TULINO A MVERDU S, "Random matrix theory and wireless communications," Communications and Information Theory, vol. 1, no. 1, pp. 1-182, (2004).

8. WEI H,WANG D M,ZHU H L,et al., "Mutual coupling calibration for multiuser massive MIMO systems," IEEE Journal on Selected Areas in Communications, vol. 15, no. 1, pp. 1-1, (2015).

9. D. Gesbert et al., "Multi-cell MIMO cooperative networks: A new look at interference," IEEE J. Sel. Areas Commun., vol. 28, no. 9, pp. 1380C1408,(Dec. 2010).

10. GAO XTUFVESSON FEDFORS O, "Massive MIMO channels- Measurements and models," Conference on SignalsSystems and Computers, pp. 280-284, (2013).

11. A. Paulraj, R. Nabar, and D. Gore, "Introduction to Space-Time Wireless Communications," Proc. of Cambridge Univ. Press, 2003, Cambridge, U.K,( 2003).

12. WANG Dongming,ZHANG Yu,WEI Hao, "An overview of transmission theory and techniques of large-scale antenna systems for $5 \mathrm{G}$ wireless communications," Science China:Information Sciences, vol. 46, no. 1,pp. 3-21, (2016).

13. VIEIRA J,MALKOWSKY S,NIEMAN K,et al., "A flexible 100-antenna testbed for massive MIMO," Proceedings of 2014 IEEE Globecom Workshop. Texas, USA:IEEE, pp. 287-293, (2014).

14. FADLALLAH Y,AISSA A,AMIS K,et al., "New iterative detector of MIMO transmission using sparse decomposition," IEEE Transactions on Vehicular Technology, vol. 68, no. 8, pp. 3458-3464, (2014).

15. JOSE JASHIKHMIN AMARZE'ITA T Let a1, "Pilot contamination and precoding in multi-cell TDD systems", IEEE Transactions on Wireless Communications, vol. 10, no. 8, pp. 2640-2651,(2011).

16. YU X,SHEN J C,ZHANG J,et al., "Alternating minimization algorithms for hybrid precoding in millimeter wave MIMO systems," JIEEE Journal of Selected Topics in Signal Processing, vol. 10, no. 3, pp. 485-500, (2016).

17. LI HLEUNG V C M., "Low complexity zero-forcing beamforming for distributed massive MIMO systems in large public venues," Journal of Communications and Networks, vol. 15, no. 4, pp. 370-382, (2013).

18. R. Wang and G. B. Giannakis, "Approaching MIMO channel capacity with soft detection based on hard sphere decoding," IEEE Trans. Commun.,vol. 54, no. 4, pp. 587-590, (2006). 